\title{
MTBP plays a crucial role in mitotic progression and chromosome segregation
}

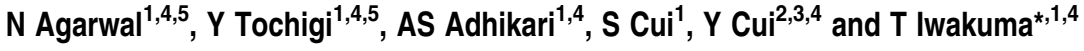

Murine double minute 2 (MDM2) binding protein (MTBP) has been implicated in tumor cell proliferation, but the underlying mechanisms remain unclear. The results of MTBP expression analysis during cell cycle progression demonstrated that MTBP protein was rapidly degraded during mitosis. Immunofluorescence studies revealed that a portion of MTBP was localized at the kinetochores during prometaphase. MTBP overexpression delayed mitotic progression from nuclear envelope breakdown (NEB) to anaphase onset and induced abnormal chromosome segregation such as lagging chromosomes, chromosome bridges, and multipolar chromosome segregation. Conversely, MTBP downmodulation caused an abbreviated metaphase and insufficient mitotic arrest, resulting in abnormal chromosome segregation, aneuploidy, decreased cell proliferation, senescence, and cell death, similar to that of Mad2 (mitotic arrest-deficient 2) downmodulation. Furthermore, MTBP downmodulation inhibited the accumulation of Mad1 and Mad2, but not BubR1 (budding uninhibited by benzimidazoles related 1), on the kinetochores, whereas MTBP overexpression inhibited the release of Mad2 from the metaphase kinetochores. These results may imply that MTBP has an important role in recruiting and/or retaining the Mad1/Mad2 complex at the kinetochores during prometaphase, but its degradation is required for silencing the mitotic checkpoint. Together, this study indicates that MTBP has a crucial role in proper mitotic progression and faithful chromosome segregation, providing new insights into regulation of the mitotic checkpoint. Cell Death and Differentiation (2011) 18, 1208-1219; doi:10.1038/cdd.2010.189; published online 28 January 2011

Murine double minute 2 (MDM2) binding protein (MTBP) was originally identified as a protein that interacts with the oncoprotein MDM2, a major negative regulator of the tumor suppressor p53. ${ }^{1}$ Overexpression of MTBP was shown to suppress cell proliferation and colony formation of several human cancer cell lines independent of their p53 status. ${ }^{1}$ Brady et $\mathrm{al}^{2}{ }^{2}$ reported that overexpression of MTBP in MCF7 cells resulted in MDM2 stabilization and subsequent p53 degradation. However, our previous findings demonstrated that complete deletion of MTBP in mice resulted in an early embryonic lethal phenotype independent of $p 53 .^{3}$ Furthermore, MTBP heterozygous mice $\left(M T B P^{+/-}\right)$were neither tumor prone nor did they show any obvious phenotypes. Nonetheless, $\mathrm{Mtbp}^{+/-} \mathrm{p} 53^{+/-}$mice showed an increased frequency of metastatic tumors compared with $p 53^{+/-}$mice, suggesting the possible involvement of MTBP in tumor progression. ${ }^{3}$ Recently, Odvody et al. $^{4}$ reported that a reduced MTBP level delayed Myc-induced lymphomagenesis independent of Mdm2 and p53. Thus, a vast majority of the results support the p53-independent functions of MTBP and its involvement in cell proliferation and tumor progression.
However, the underlying mechanisms for cellular phenotypes associated with altered MTBP expression remain unsolved.

Cell cycle progression is tightly regulated by proteins involved in cell cycle checkpoints. The mitotic checkpoint (mitotic spindle assembly checkpoint), which regulates proper mitotic cell division to ensure faithful chromosome segregation, monitors chromosome alignment during mitosis by delaying metaphase-anaphase transition until all chromosomes are properly attached to the mitotic spindle and aligned at the metaphase plate. ${ }^{5}$ The mitotic checkpoint signal to the cell is relayed by kinetochores, the structures formed at centromeres, during mitosis for binding microtubules. Lack of kinetochore-microtubule attachment or loss of tension on the kinetochore microtubules activates the mitotic checkpoint via recruitment of several spindle checkpoint proteins, including Mad1 (mitotic arrest-deficient 1), Mad2, Bub1 (budding uninhibited by benzimidazole 1), Bub3, MPS1 (mono polar spindle 1), CENP-E, and BubR1. ${ }^{6}$ Recruitment of these proteins to the kinetochores promotes the formation of a mitotic checkpoint complex (MCC), which contains Mad2, BubR1, Bub3, and cell division cycle 20 (Cdc20). The MCC

\footnotetext{
${ }^{1}$ Department of Genetics, Louisiana State University Health Sciences Center, 533 Bolivar Street, CSRB, Room 439, New Orleans, LA 70112, USA; ${ }^{2}$ Department of Medicine, Louisiana State University Health Sciences Center, 533 Bolivar Street, CSRB, Room 439, New Orleans, LA 70112, USA; ${ }^{3}$ Gene Therapy Center, Louisiana State University Health Sciences Center, 533 Bolivar Street, CSRB, Room 439, New Orleans, LA 70112, USA and ${ }^{4}$ Stanley S. Scott Cancer Center, Louisiana State University Health Sciences Center, 533 Bolivar Street, CSRB, Room 439, New Orleans, LA 70112, USA

${ }^{*}$ Corresponding author: T Iwakuma, Department of Genetics, Louisiana State University Health Sciences Center, 533 Bolivar Street, CSRB, Room 439, New Orleans, LA 70112, USA. Tel: 504-568-3235; Fax: 504-568-8500; E-mail: tiwaku@Isuhsc.edu

${ }^{5}$ These authors contributed equally to this work.

Keywords: MTBP; MDM2; mitosis; checkpoint; chromosome segregation

Abbreviations: MDM2, murine double minute 2; BubR1, budding uninhibited by benzimidazoles related 1; Mad, mitotic arrest-deficient; Cdc20, cell division cycle 20; MPS1, mono polar spindle 1; PBS, phosphate-buffered saline; DMSO, dimethyl sulfoxide; DAPI, 4',6-diamidino-2-phenylindole; SDS, sodium dodecyl sulfate; NEB, nuclear envelope breakdown; MTBP, MDM2 binding protein; MCC, mitotic checkpoint complex; APC/C, anaphase-promoting complex/cyclosome; CHX, cycloheximide; PI, propidium iodide; MOI, multiplicity of infection; SA, senescence associated; ATCC, American Type Culture Collection; PMSF, phenylmethylsulfonyl fluoride; TBS, Tris-buffered saline; TBS-T, TBS with $0.1 \%$ Tween-20

Received 17.8.10; revised 20.12.10; accepted 20.12.10; Edited by V De Laurenzi; published online 28.1.11
} 
inhibits the function of the anaphase-promoting complex/ cyclosome $(\mathrm{APC} / \mathrm{C})$ that degrades both cyclin B and securin via the ubiquitin-dependent pathway, thereby regulating anaphase onset. ${ }^{5}$ Cells with a partial or complete loss of the aforementioned crucial molecules of the mitotic checkpoint manifest insufficient mitotic arrest, chromosome missegregation, abnormal DNA ploidy, senescence, cell death, and cellular transformation. ${ }^{7-10}$ Intriguingly, an overactive mitotic checkpoint also leads to mitotic cell death, chromosome missegregation, and aneuploidy. ${ }^{11-14}$

To illustrate the participation of MTBP in cell cycle regulation and to understand the underlying molecular mechanism, we examined the effects of modulating MTBP expression on cell cycle progression. We found that MTBP overexpression resulted in delayed mitotic progression with abnormal chromosome segregation, whereas MTBP downmodulation caused an abbreviated metaphase and insufficient mitotic arrest, consequently leading to abnormal chromosome segregation and decreased cell proliferation with cell death, aneuploidy, and senescence. Thus, our findings suggest that MTBP has a crucial role in proper mitotic progression and chromosome segregation.

\section{Results}

Expression and localization of MTBP during mitosis. To understand the physiological role of MTBP in cell cycle regulation, we examined the kinetics of endogenous MTBP expression during cell cycle progression using cyclin $A$ and cyclin B1 as markers (Figures 1a and b). To synchronize HeLa cells at the early $\mathrm{G} 1$ phase of the cell cycle, we treated them with mimosine and examined the expression levels of endogenous MTBP, cyclin A, cyclin B1, and $\beta$-tubulin at various time points following their release from early $\mathrm{G} 1$ arrest. The levels of endogenous MTBP protein gradually increased as the cells progressed from early $\mathrm{G} 1$ to $\mathrm{G} 2 / \mathrm{M}$ phases of the cell cycle, similar to that of cyclin B1 (Figure 1a). To examine the expression of MTBP during mitotic progression, we treated HeLa cells with nocodazole to arrest them at prometaphase and harvested cells for western blotting at various time points following their release from nocodazole block. Similar to that of cyclin B1, the endogenous MTBP protein levels were rapidly attenuated during mitotic progression (Figure 1b). To determine if the attenuation of MTBP protein expression occurred at the transcriptional or post-translational levels, we examined mRNA expression and MTBP protein stability following nocodazole release. No significant change in MTBP mRNA levels was observed within $4 \mathrm{~h}$ after nocodazole release, whereas endogenous MTBP protein levels were rapidly reduced in the presence of cycloheximide ( $\mathrm{CHX}$ ), similar to cyclin B1 (Figures 1c and d). Hence, we concluded that rapid reduction of MTBP protein levels during mitotic progression was mediated through its degradation.

To directly visualize the mitotic phase-specific degradation and cellular localization of MTBP, we performed immunofluorescence staining for endogenous MTBP and DNA in HeLa cells (Figure 1e). Approximately $90 \%$ of cells during prophase to prometaphase showed medium to high signal intensities for MTBP. However, the intensities were markedly reduced during metaphase, as $\sim 60 \%$ cells from metaphase to telophase displayed low or undetectable signals for MTBP (Figure 1e). Notably, signals for MTBP overlapped with chromatin stained with $4^{\prime}, 6$-diamidino-2-phenylindole (DAPI) till prophase, but the majority of MTBP no longer overlapped with chromatin in cells during prometaphase (Figure 1e). Thus, MTBP degradation occurred during metaphase following its cellular translocation at prometaphase. Intriguingly, we found that a portion of MTBP was localized at the kinetochores during prometaphase, as MTBP staining overlapped with that by the anticentromere antibody CREST (Figure 1f). During metaphase, MTBP signals were much less when compared with those observed during prometaphase (Supplementary Figure S1). These dynamic changes in the expression and cellular localization of MTBP during mitosis suggest its potential role in the regulation of mitotic progression.

Overexpression of MTBP delays mitotic progression. To examine the effect of MTBP overexpression on cell cycle progression, we transduced HeLa cells with either MTBPencoding or control adenoviral vectors, and performed propidium iodide (PI) staining and flow cytometric analyses. Our titration experiments of adenoviral vectors in HeLa cells demonstrated a dose-dependent increase in G2/M phase of the cell cycle with increased MTBP levels (Supplementary Figure $\mathrm{S} 2 \mathrm{a}$, left). Use of 20 multiplicities of infection (MOI) of the MTBP-adenoviral vector resulted in $\sim 13$-fold higher levels of MTBP than endogenous MTBP (0 MOI, Supplementary Figure S2a, right). As $20 \mathrm{MOls}$ of the control vector did not alter the cell cycle profile, we used this titer throughout experiments. In all cell lines examined, including HeLa, HCT116 p53 ${ }^{+/+}$, and HCT116 p53 ${ }^{-/-}$cells, MTBP overexpression resulted in a modest accumulation of cells at the G2/M phase of the cell cycle compared with control cells (Figure 2a), suggesting a p53-independent role of MTBP in mitotic progression. To further examine the effect of MTBP overexpression on mitotic progression, we treated HeLa cells with nocodazole and performed cell cycle profile analyses at various time points after releasing cells from nocodazole block. MTBP overexpression delayed cell cycle progression from G2/M to G1 phases when compared with control cells (Figure 2b, left). Western blotting revealed that exogenous MTBP was also attenuated with time after release from nocodazole block similarly to endogenous MTBP, but was retained at a higher level of expression during the course of the experiment (Figure 2b, right).

To determine the specific mitotic phase affected by MTBP overexpression, we performed time-lapse microscopy using HeLa cells stably expressing histone H2B fused to a monomeric cherry fluorescent protein (histone H2B-mCherry). MTBP overexpression significantly delayed the duration of nuclear envelope breakdown (NEB) to anaphase onset, specifically of either prometaphase or metaphase (Figure 2c and Supplementary Figure S2b). Approximately 60\% (21 out of 35) of cells with MTBP overexpression showed a prolonged prometaphase (>25 min) or metaphase (>40 min), whereas only $11 \%$ (3 out of 27 ) of control cells showed these abnormal phenotypes (Supplementary Figure S2b). Interestingly, cells 
a

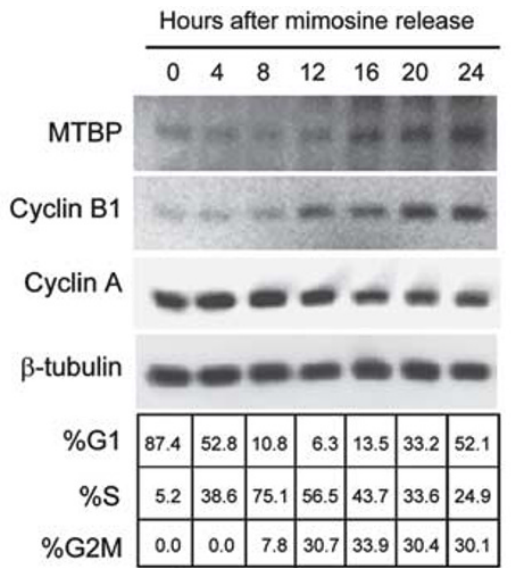

b

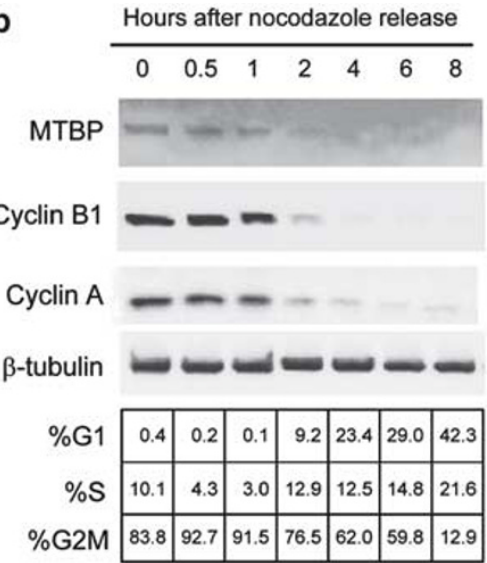

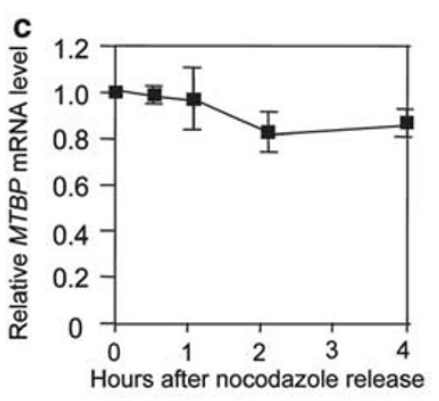

d

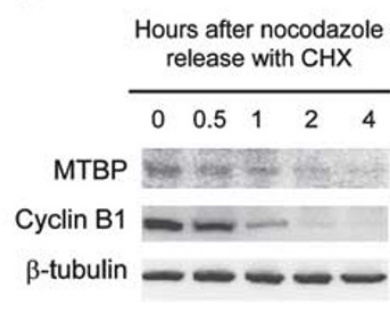

e
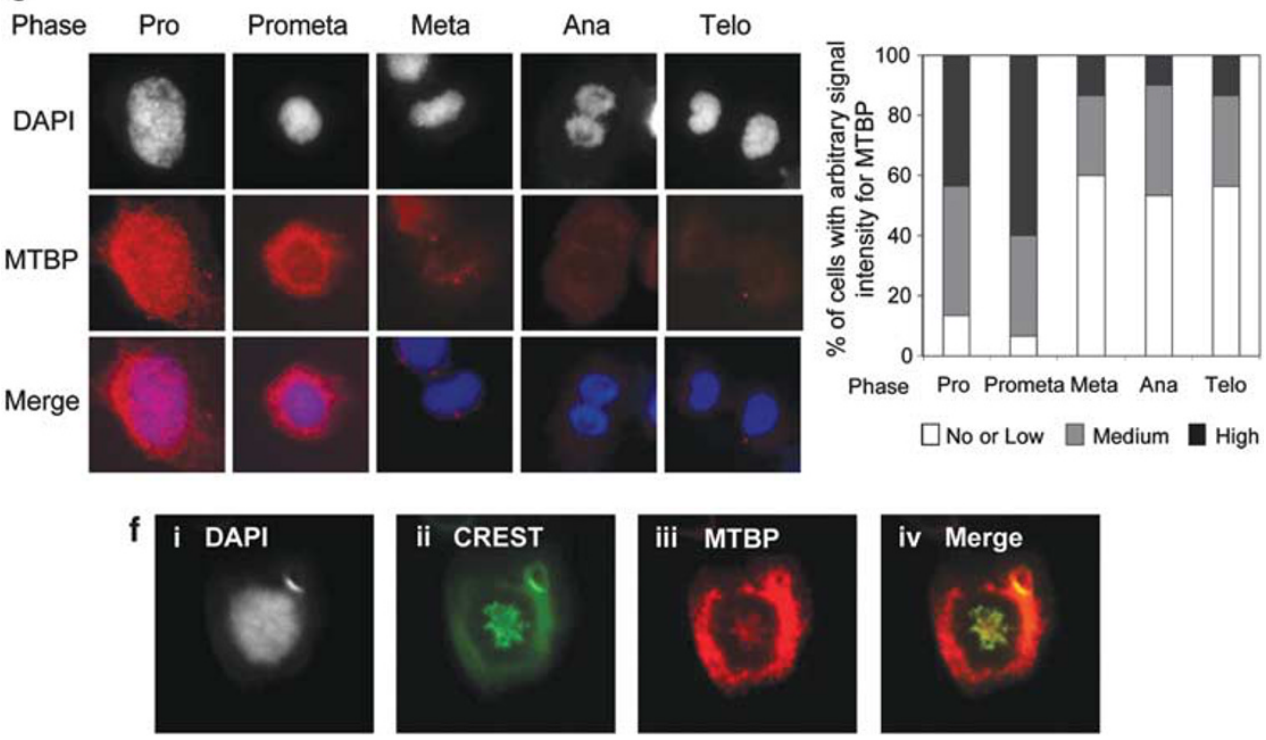

Figure 1 Expression and localization of MTBP during mitosis. (a and $\mathbf{b}$ ) HeLa cells synchronized at the G1 phase of the cell cycle with mimosine (a) or at the prometaphase using nocodazole (b) were reseeded and harvested at the indicated time points for western blotting of endogenous protein expression of MTBP, cyclin B1, cyclin A, and $\beta$-tubulin. The rest of the cells were used to examine cell cycle profiles. (c) MTBP mRNA expression was measured by quantitative real-time PCR. Relative mRNA expression was normalized to the value of Gapdh. Average values with S.D. from three independent experiments. (d) Endogenous protein stabilities for MTBP and cyclin B1 during mitosis were measured in the presence of cycloheximide (CHX) following nocodazole release of HeLa cells. Representative western blotting image (left) and quantification by densitometric analysis of MTBP and cyclin B1 (right). (e) HeLa cells were treated with nocodazole for $15 \mathrm{~h}$ and released from the treatment for $5 \mathrm{~h}$ to enrich mitotic cell population. Both attached and unattached cells were fixed and stained for DNA (DAPI, blue), and MTBP (red). Representative immunofluorescence results in each mitotic phase (left). A total of 30 cells in each mitotic phase were examined under fluorescence microscope for MTBP expression. Cells were arbitrarily scored on the basis of signal intensity for MTBP as high, medium, and low or no signal (right). The percentages of cells in each group were determined. (f) Immunofluorescence study for DNA (i), centromere (CREST, ii), and endogenous MTBP (iii) during prometaphase. Images were obtained on a Zeiss Axioplan II deconvolution microscope (Carl Zeiss). Representative Z-sectioned images are shown. A merged picture (iv) of the staining results for CREST and MTBP 
a

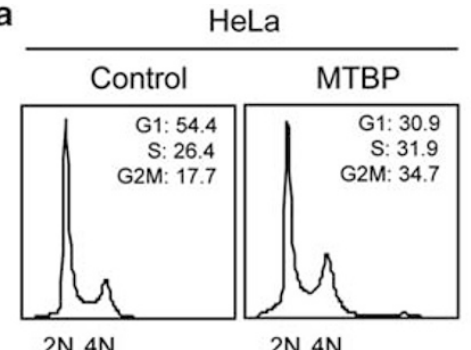

HCT116 p53+/+

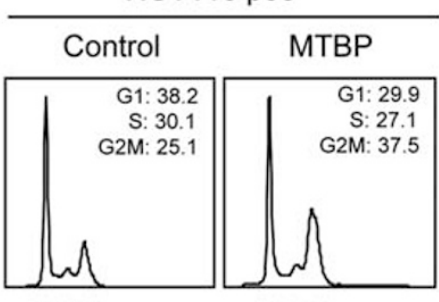

$2 \mathrm{~N} 4 \mathrm{~N}$
$2 \mathrm{~N} 4 \mathrm{~N}$
HCT116 p53\%

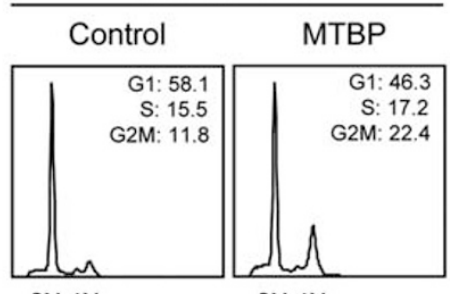

$2 \mathrm{~N} 4 \mathrm{~N}$

b

Hours after nocodazole release

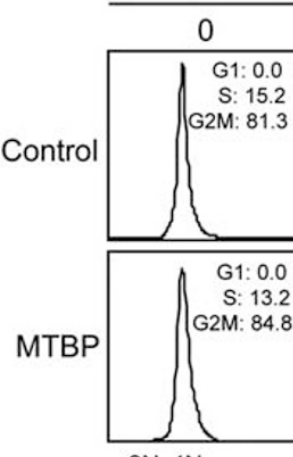

$$
2
$$

4

6

\begin{tabular}{r||r|} 
G1: 39.4 & G1: 48.5 \\
S: 16.3 & S: 13.6 \\
$2 M: 42.4$ & G2M: 31.6
\end{tabular}

Control

MTBP

Hours after nocodazole release \begin{tabular}{llllllllll}
\hline 0 & 1 & 2 & 4 & 6 & 0 & 1 & 2 & 4 & 6
\end{tabular}

MTBP $\beta$-tubulin - - - - - - $2 \mathrm{~N} 4 \mathrm{~N}$ $2 \mathrm{~N} 4 \mathrm{~N}$ $2 \mathrm{~N} 4 \mathrm{~N}$ $2 \mathrm{~N} 4 \mathrm{~N}$
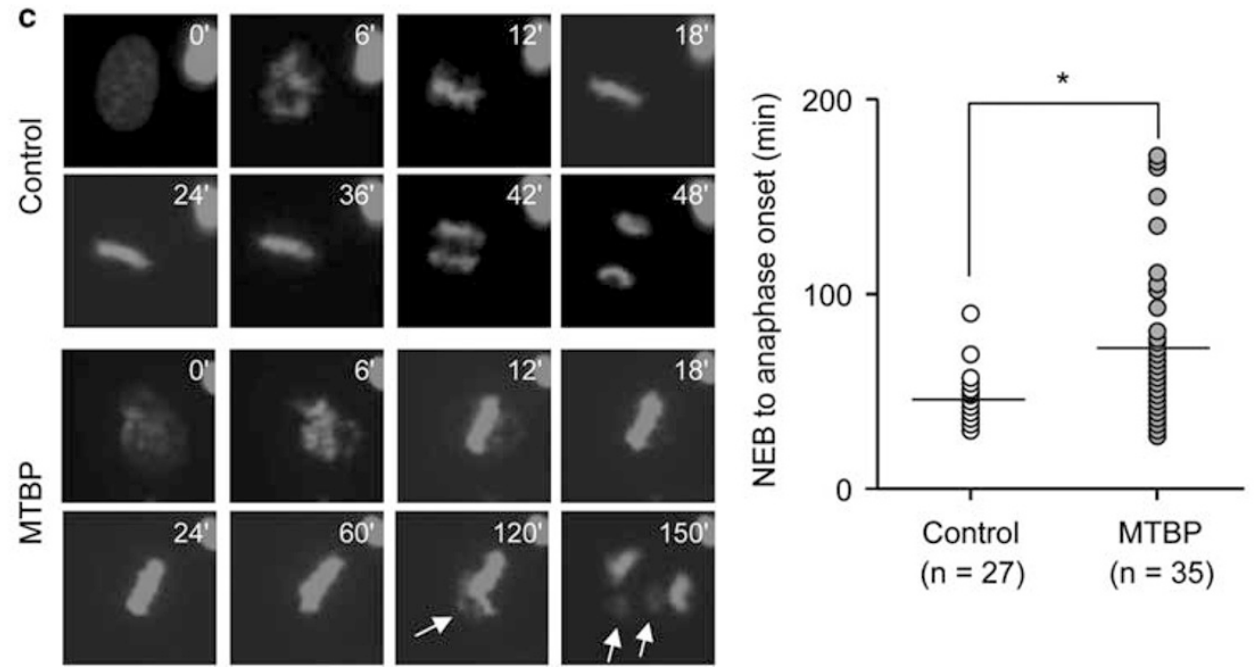

Figure 2 MTBP overexpression results in the accumulation of cells at G2/M phase of the cell cycle because of delayed mitotic progression. (a) At $48 \mathrm{~h}$ after infection of exponentially growing HeLa, HCT116 p53 ${ }^{+/+}$, and HCT116 p53 $3^{-/-}$cells with $20 \mathrm{MOls}$ of control empty (control) or MTBP-encoding (MTBP) adenoviral vectors, the cells were examined for cell cycle profiles by flow cytometry. (b) HeLa cells were infected with control empty or MTBP-encoding adenoviral vectors (20 MOls). After $48 \mathrm{~h}$, cells were treated with nocodazole for $24 \mathrm{~h}$. Mitotic cells were harvested using the mitotic shake-off method. Flow cytometry (left) and western blotting (right) were performed at the indicated time points following release from nocodazole block. (c) Time-lapse microscopy using HeLa cells stably expressing histone H2B-mCherry. At $48 \mathrm{~h}$ after transfection with a plasmid encoding MTBP fused to T2A self-cleaving peptide-copGFP (System Biosciences, Mountain View, CA, USA), only cells expressing GFP were processed for time-lapse microscopy analysis. Images were acquired every $3 \mathrm{~min}$ and analyzed for mitotic progression and duration of different mitotic phases. Arrows indicate lagging chromosomes. Duration from NEB to anaphase onset is shown as a graph (right, ${ }^{*} P<0.01$ )

with a prolonged prometaphase conversely had an abbreviated metaphase, which was $<3 \mathrm{~min}$. We also found that $\sim 30 \%$ of MTBP overexpressed cells in anaphase showed abnormal chromosome segregation, such as lagging chromosomes, chromosome bridges, and multipolar chromosome segregation (Figure 2c, arrows). These results suggest a crucial role of MTBP in mitotic progression and chromosome segregation.
MTBP downmodulation abbreviated metaphase duration and induced chromosome missegregation. Faithful chromosome segregation is governed by the mitotic checkpoint. Therefore, loss of mitotic checkpoint control results in abnormal chromosome segregation. To examine if MTBP downmodulation also leads to chromosome missegregation via impairment of the mitotic checkpoint, we performed immunofluorescence staining for DNA and $\alpha$-tubulin after 
transfection of control siRNA and two different MTBP siRNAs (MTBP\#1 and MTBP\#2) into HeLa cells. We used siRNAs for Mad2 and BubR1 as positive controls for impaired mitotic checkpoint and chromosome missegregation. Western blotting results confirmed that all siRNAs successfully downmodulated the corresponding protein expression (Supplementary Figure S3a). Approximately $30-35 \%$ of the MTBP downmodulated cells displayed abnormal chromosome segregation such as misaligned chromosomes, chromosome bridges, lagging chromosomes, and multipolar chromosome segregation, similar to Mad2 and BubR1 downmodulated cells (Figure 3a). In contrast, only $7 \%$ of control cells showed these phenotypes. Interestingly, MTBP downmodulation generated more multipolar chromosome segregation and less lagging chromosomes and chromosome bridges than Mad2 and BubR1 downmodulation.

To further determine the specific mitotic phase affected by MTBP downmodulation, we performed time-lapse microscopy following downmodulation of MTBP in HeLa cells stably expressing histone H2B-eGFP (Figure $3 b$ ). Cells with MTBP downmodulation showed a significant reduction of metaphase duration compared with that of control cells (Figure $3 b$ ). Approximately $30 \%$ of cells with MTBP downmodulation and $80 \%$ of the Mad2 downmodulated cells had $<3$ min of metaphase, whereas only $8 \%$ of control cells showed the abbreviated metaphase (Figure $3 \mathrm{~b}$ ). We also detected abnormal chromosome segregation similar to the result of Figure 3a. Other mitotic phases were not affected (data not shown). Similar results were obtained using HCT116 cells (Supplementary Figure S3b). Approximately $80 \%$ of HCT116 cells with MTBP downmodulation and $70 \%$ of Mad2 downmodulated cells had $<3$ min of metaphase, whereas only $21 \%$ of control cells showed the abbreviated metaphase. These results suggest that MTBP has a crucial role in faithful chromosome segregation and mitotic checkpoint regulation.

Cells with downmodulation of proteins involved in the mitotic checkpoint manifest insufficient mitotic arrest and therefore a reduced mitotic index upon exposure to nocodazole, a microtubule depolymerizing agent. ${ }^{7,8,15}$ To confirm the involvement of MTBP in the mitotic checkpoint, we examined the percentage of mitotic cells (mitotic index) following treatment of cells with nocodazole or dimethyl sulfoxide (DMSO; Figure 3c). We measured the mitotic index via flow cytometry using a histone H3 S10P antibody in combination with DNA content analysis. ${ }^{16}$ Following nocodazole treatment, MTBP downmodulation significantly reduced the percentage of mitotic cells compared with the control, but to a lesser extent than those by Mad2 and BubR1 downmodulation (Figure 3c, Control: $39 \pm 4 \%$, MTBP\#1: $21 \pm 6 \%$, MTBP\#2: $23 \pm 4 \%$, Mad2: $3 \pm 1 \%$, BubR1: $3 \pm 1 \%$ ). Thus, deletion of MTBP expression resulted in insufficient mitotic arrest, indicating the positive involvement of MTBP in the mitotic checkpoint. We further demonstrated that exogenous FLAG-MTBP partially, but considerably, rescued the reduced mitotic index by MTBP downmodulation. This suggests that the FLAG-tagged MTBP functions similar to endogenous MTBP (Supplementary Figure S3c). Observed partial rescue may be attributed to the difference in the mechanism by which MTBP downmodulation and its overexpression affect mitotic progression.
MTBP downmodulation resulted in decreased cell proliferation with an increase in cellular senescence, cell death, and aneuploidy. Insufficient mitotic arrest results in abnormal cell cycle progression and decreased cell proliferation via chromosome abnormality. ${ }^{12,17-19}$ Hence, we wanted to examine the long-term consequences of MTBP downmodulation on cellular behavior and chromosome aneuploidy. We first examined the effect of MTBP downmodulation on cell growth using colony outgrowth assays (Figure 4a). HeLa cells transfected with the same set of siRNAs as in Figure 3 were treated with nocodazole or DMSO. Both attached and floating cells were harvested and washed with phosphate-buffered saline (PBS) to release them from nocodazole block. Only live cells were examined for their ability to form colonies in regular medium. Cells transfected with control siRNA formed similar numbers of colonies regardless of DMSO or nocodazole block and release. Cells with MTBP, Mad2, and BubR1 downmodulation showed significantly reduced colony formation compared with the control even without exposure to nocodazole. Upon exposure to nocodazole, MTBP downmodulated cells showed few or no colony formation, similarly to Mad2 and BubR1 downmodulation (Figure 4a).

We next performed cell proliferation assays (Figure 4b). At 4 days after transfection (2 days after reseeding), MTBP downmodulated HeLa cells started showing reduced cell proliferation similar to the cases of Mad2 and BubR1 downmodulation (Figure 4b). We also obtained similar results using HCT116 cells (Supplementary Figure S4a). Interestingly, at 9 to 11 days after siRNA transfection, some cells transfected with MTBP, Mad2, and BubR1 siRNAs showed senescence-like cellular phenotypes, such as an enlarged cell size, flattened cell morphology, and vacuolated cell forms (Supplementary Figure S4b). We therefore employed senescence-associated (SA)- $\beta$-gal staining of cells (Figure $4 \mathrm{c}$ ). As expected, a significantly higher percentage of cells with MTBP, Mad2, and BubR1 downmodulation showed positive SA- $\beta$-gal staining compared with control cells (Figure $4 \mathrm{c}$ and Supplementary Figure S4b).

In order to examine the effects of MTBP downmodulation on cell cycle profile and aneuploidy, we further performed PI staining and flow cytometric analyses (Figure 4d). Cells were processed 4 days after siRNA transfection, as differences in cell proliferation started appearing around that time. MTBP downmodulation increased the cell population in the sub-G1, $\mathrm{G} 2 / \mathrm{M}$, and super-G2/M phases of the cell cycle. There was also an increase in aneuploid and polyploid cells, similar to Mad2 downmodulation (Figure 4d). These results suggest that MTBP downmodulation induced abnormal chromosome segregation and cell death, indicating that MTBP has the crucial role in proper mitotic progression and faithful chromosome segregation.

MTBP downmodulation inhibits the accumulation of Mad1 and Mad2, but not BubR1, on the kinetochores. The crucial proteins involved in the mitotic checkpoint, such as Mad2 and BubR1, are recruited to the kinetochores to assemble the MCC during prometaphase. To better understand how MTBP participates in the mitotic checkpoint regulation, we examined the effect of MTBP downmodulation 
a
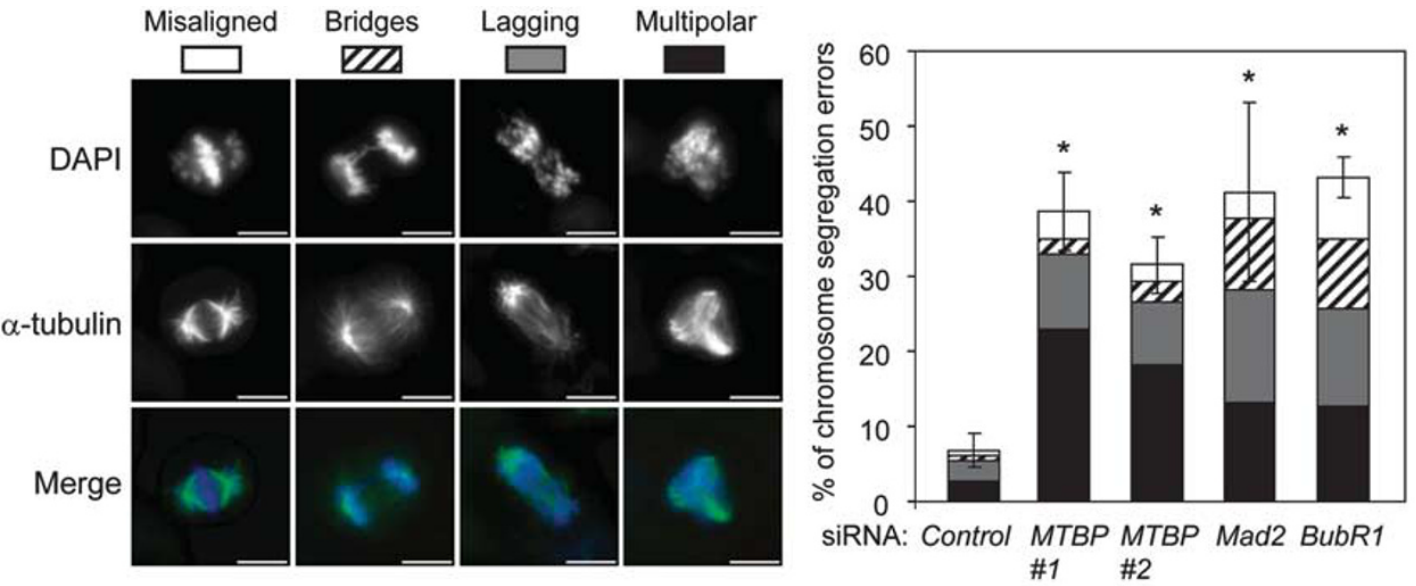

b
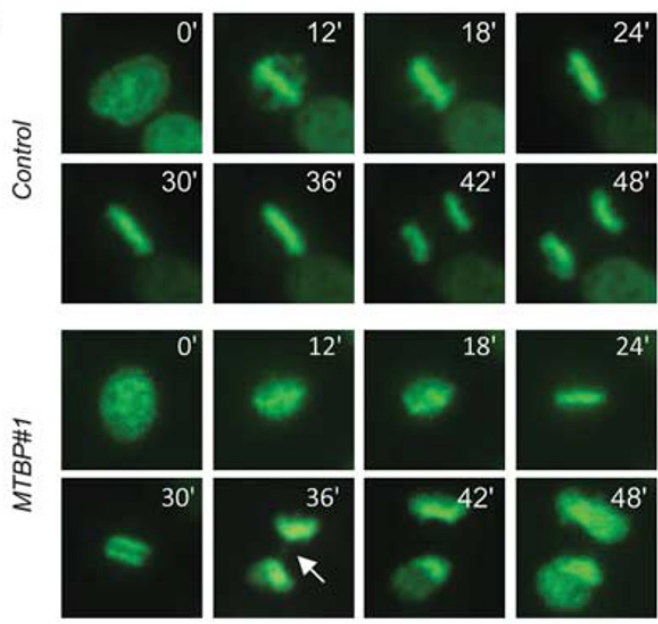

C

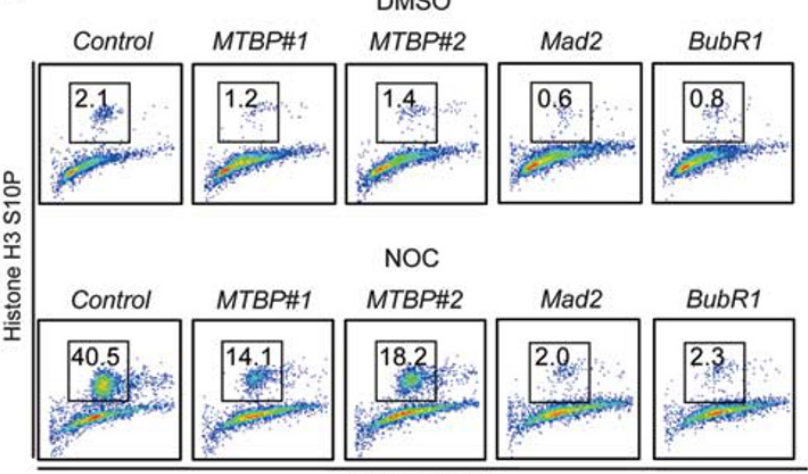

DNA content
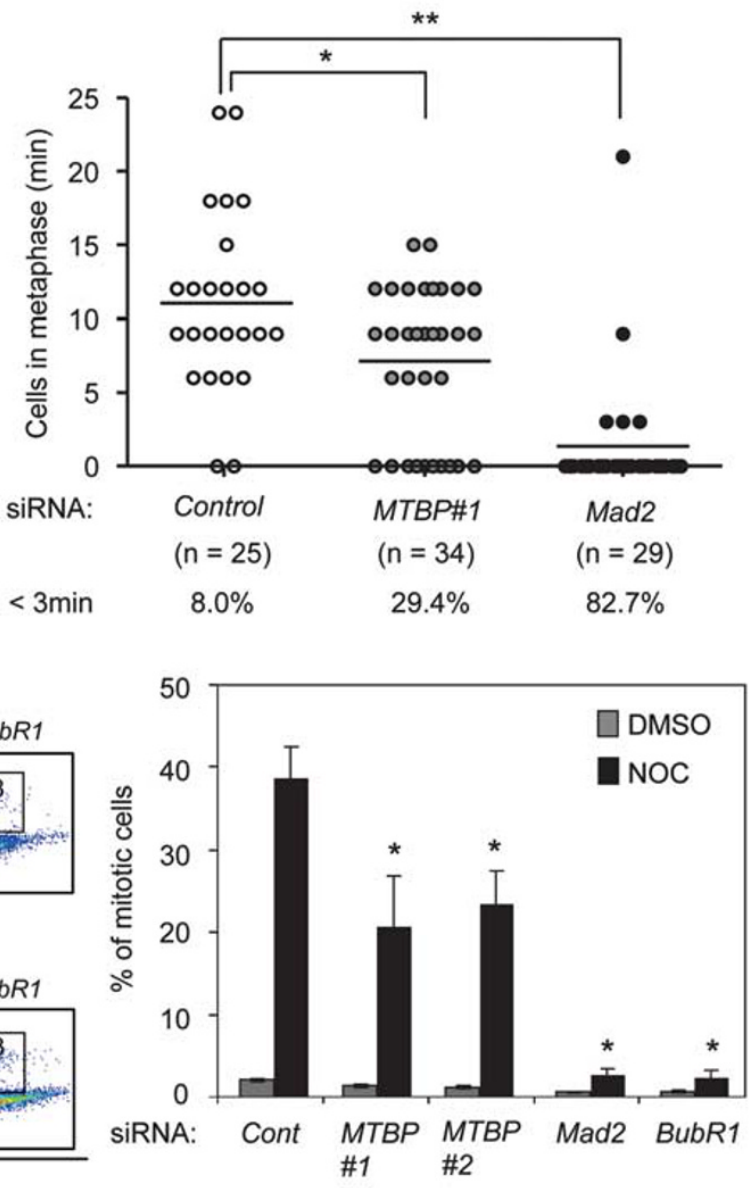

Figure 3 MTBP downmodulation results in chromosome missegregation and insufficient mitotic arrest. (a) HeLa cells transfected with the indicated siRNAs were stained for DNA (DAPI, blue) and $\alpha$-tubulin (green). Cells solely in the mitotic phase were examined for the chromosome abnormalities indicated $(n=100)$, and percentages of cells with chromosome missegregation were determined. Representative pictures from cells with MTBP downmodulation (left) and a graph from three independent experiments (right, mean \pm S.D., ${ }^{*} P<0.01$ ). Scale bar: $10 \mu \mathrm{m}$. (b) Time-lapse microscopy following MTBP downmodulation in HeLa cells. At $48 \mathrm{~h}$ after transfection with $M T B P$, Mad2, or control siRNAs, HeLa cells stably expressing histone H2B-eGFP were processed for time-lapse imaging. Images were acquired every 3 min and analyzed for mitotic progression. An arrow indicates chromosome bridge. Graph showing duration of metaphase (right, mean, ${ }^{*} P<0.05,{ }^{* \star} P<0.01$ ). (c) HeLa cells transfected with the indicated siRNAs were treated with DMSO or nocodazole (NOC) for $24 \mathrm{~h}$ and then measured for the mitotic index. Representative results of flow cytometric analyses with percentages of phospho-histone H3 serine 10 (Histone H3 S10P)-positive mitotic cells in squares (left) and graph from three independent experiments (right, mean \pm S.D., ${ }^{\star} P<0.01$ )

on the kinetochore localization of Mad2 and BubR1 in HeLa cells following nocodazole treatment. MTBP downmodulation resulted in impaired accumulation of Mad2 on the kinetochores that were identified by the anticentromere antibody CREST (Figure 5a). Kinetochore accumulation of BubR1 was unaffected (Figure 5b). 
a

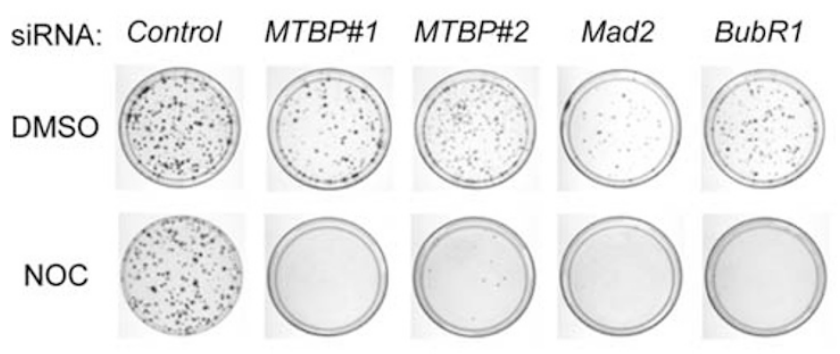

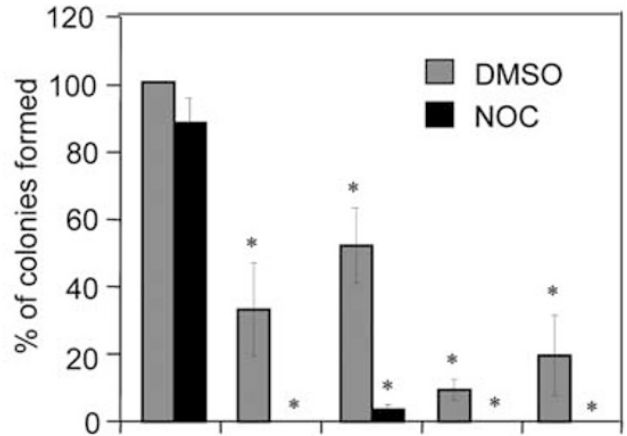

siRNA: Control MTBP MTBP Mad2 BubR1 \#1

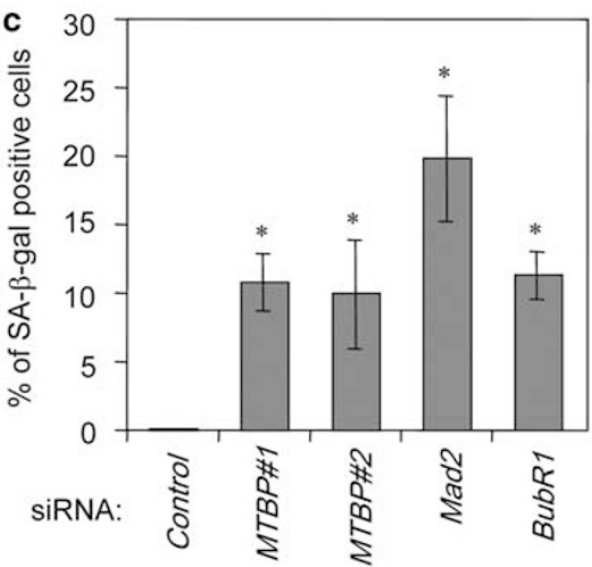

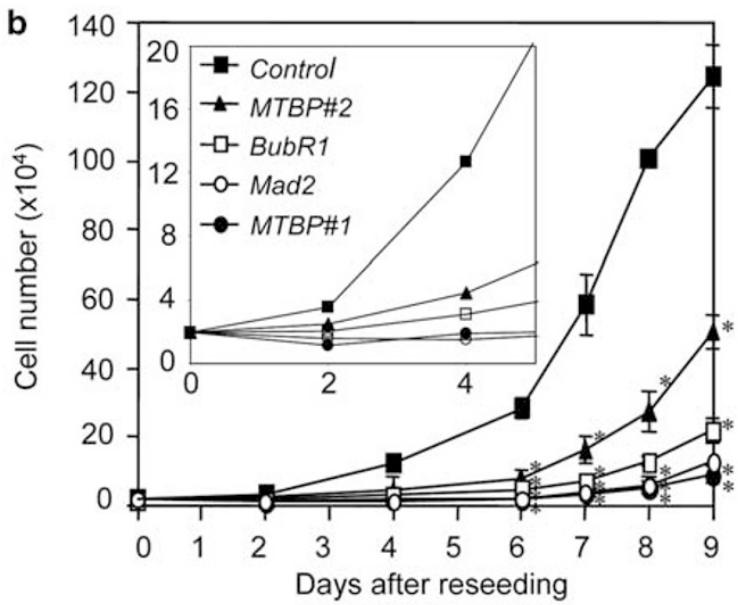

d

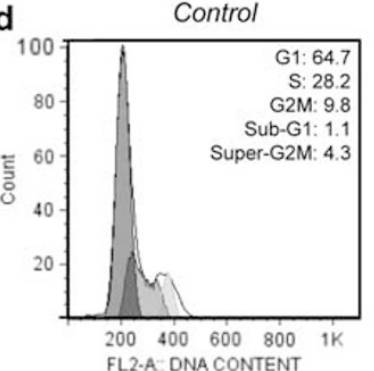

MTBP\#1

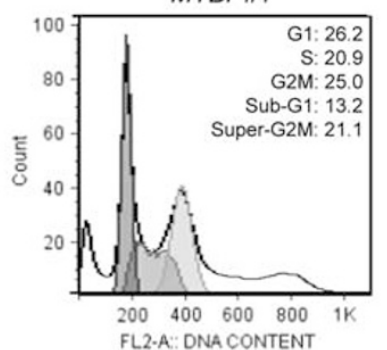

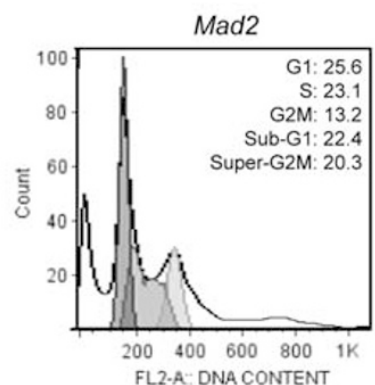

Figure 4 MTBP downmodulated cells have cellular phenotypes similar to those with downmodulation of Mad2 and BubR1. (a) Following transfection of the indicated siRNAs and subsequent treatment with DMSO or NOC, both attached and floating cells were harvested and were washed with PBS to release them from nocodazole block. Only live cells (2000) using Trypan blue staining were subjected to colony outgrowth assays. Representative colonies (left) and graph (right). The percentages of colonies formed were calculated as relative numbers normalized against that of control siRNA-transfected cells treated with DMSO (mean \pm S.D., ${ }^{*} P<0.01$ ). (b) At $48 \mathrm{~h}$ after siRNAs transfection into HeLa cells, cells $\left(2 \times 10^{4}\right)$ were re-seeded onto six-well plates in triplicate (day 0). Cell numbers were counted at the indicated days after reseeding (mean \pm S.D., ${ }^{*} P<0.01$ ). (c) HeLa cells transfected with the indicated siRNAs were reseeded onto 24-well plates. After 7 days, cells were subjected to senescenceassociated (SA)- $\beta$-gal staining. The percentages of SA- $\beta$-gal staining-positive cells $(n=100)$ are shown in the graph. Statistical significance was calculated by comparing each group with the result of control siRNA transfection (mean \pm S.D., ${ }^{\star} P<0.01$ ). Representative pictures are shown in Supplementary Figure S4b. (d) Representative results of flow cytometric analyses at 4 days following transfection of control, MTBP\#1, and Mad2 siRNAs

Mad2 forms a complex with Mad1 during interphase in order to be located at the kinetochores during prometaphase. ${ }^{20,21}$ We therefore examined the effect of MTBP downmodulation on the kinetochore localization of Mad1, and found that MTBP downmodulation also inhibited the accumulation of Mad1 on the kinetochores (Figure $5 \mathrm{c}$ ). Similar results were obtained using HCT116 cells (Supplementary Figure S5a and $b$ ). These results suggest that MTBP is required for the accumulation of Mad1 and Mad2 on the kinetochores, and it may affect mitotic checkpoint activity via regulation of Mad1/Mad2 function. We next examined if MTBP affected either the expression levels or interactions of Mad1 and Mad2. However, MTBP downmodulation neither altered the expression levels of these proteins nor their interactions in both HeLa and HCT116 cells (Supplementary Figure 5c). 
a
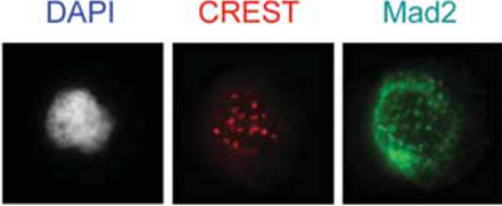

MTBP\#1
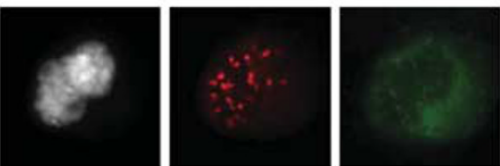

Mad2
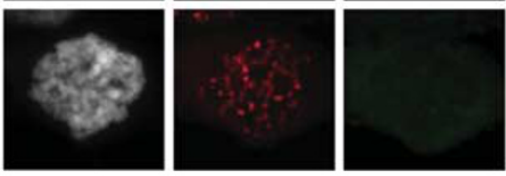

b
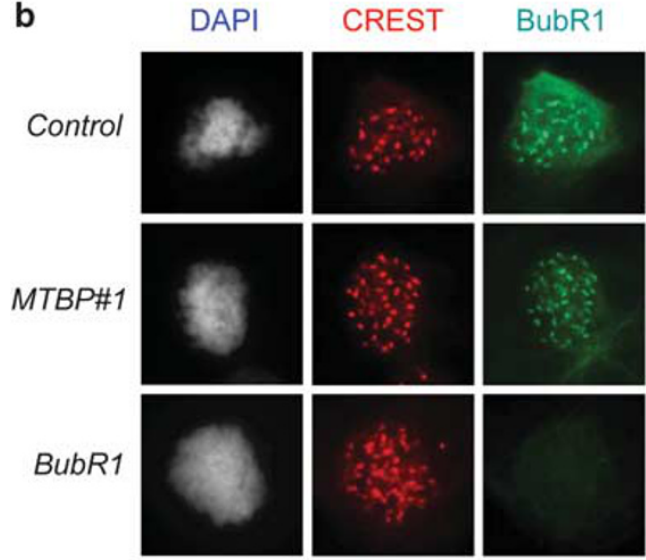

C

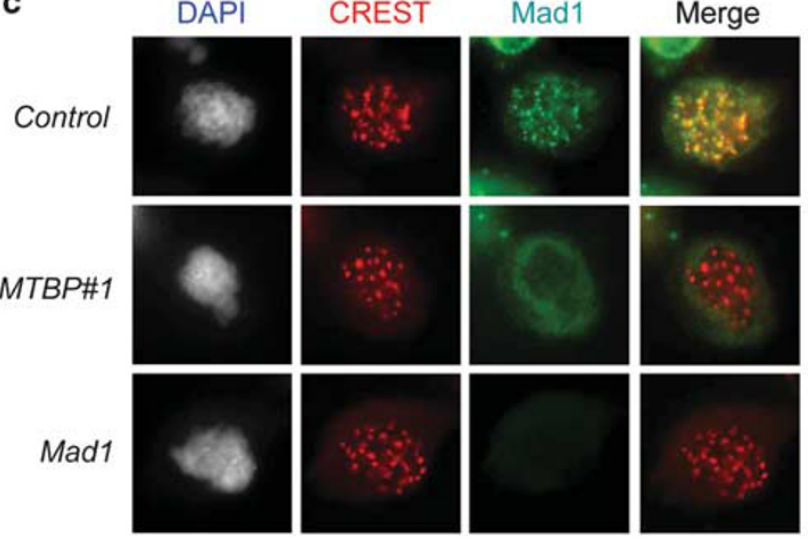

Merge

Merge

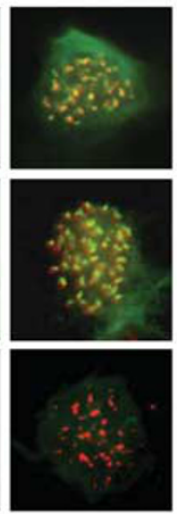

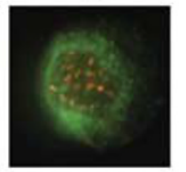
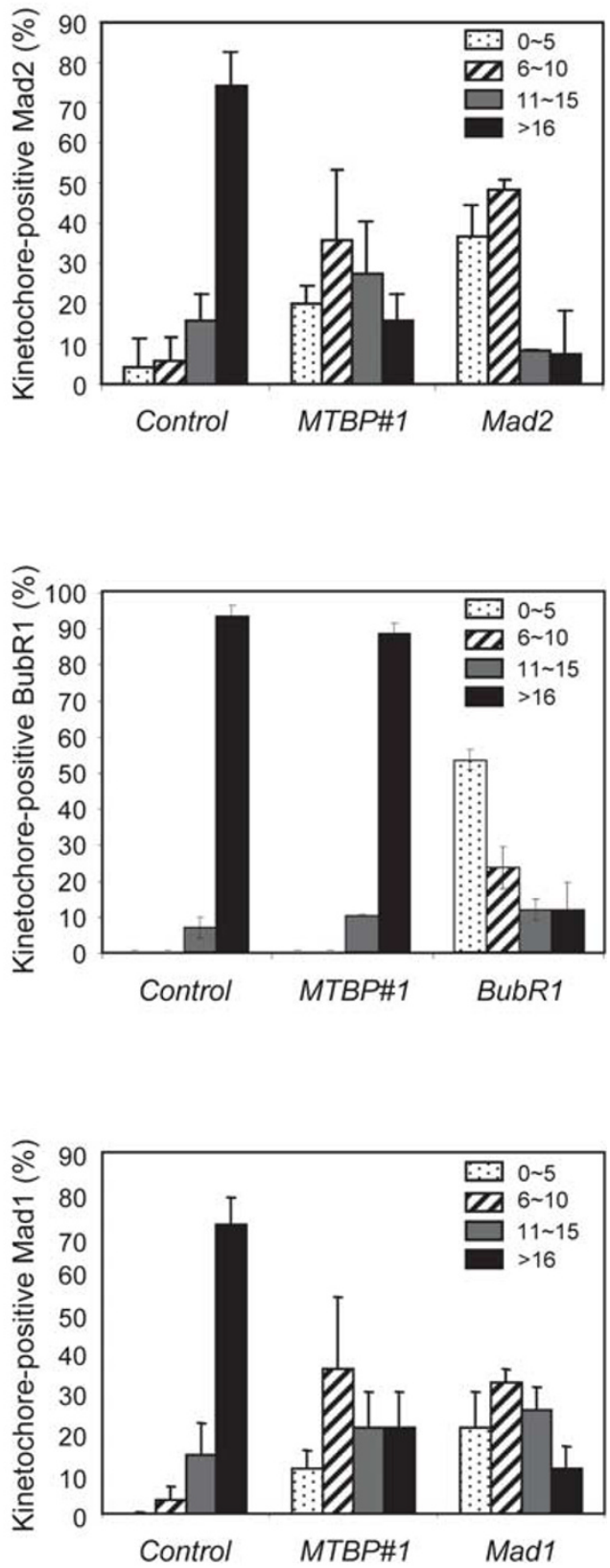

Figure 5 Downmodulation of MTBP inhibits the accumulation of Mad1 and Mad2, but not BubR1, on the kinetochores. (a-c) HeLa cells transfected with the indicated siRNAs were treated with nocodazole for $24 \mathrm{~h}$ and stained for DNA (DAPI, blue), centromere (CREST, red), and Mad2 (a, green), BubR1 (b, green), or Mad1 (c, green). Cells $(n=50)$ in prometaphase were examined for the kinetochore localization of Mad2 (a), BubR1 (b), or Mad1 (c) under a fluorescent microscope. Based on the numbers of Mad2, BubR1, or Mad1 foci at the kinetochores (indicated by CREST staining), cells were categorized into 0-5, 6-10,11-15, and >16. Percentages of cells in each category are shown. Representative results (left) and graphs from three independent experiments (right, mean \pm S.D). Merged pictures of the staining results for CREST and Mad2 (a), BubR1 (b), or Mad1 (c)

MTBP affects mitotic checkpoint function via the Mad2 pathway. To further understand the genetic and functional association of MTBP with mitotic checkpoint function, specifically with the Mad2 pathway, we wanted to compare the mitotic index of cells doubly downmodulated for MTBP and Mad2 to that with Mad2 downmodulation alone
(Figure 6a). For this, we first performed siRNA titration experiments (Supplementary Figure S6). Mitotic indices of cells with MTBP or Mad2 downmodulation were reduced in a dose-dependent manner. We chose 2 and $6 \mathrm{nM}$ of Mad2 and MTBP siRNAs, respectively, as these doses reduced mitotic index to a degree that allowed us to find an epistatic effect 
a

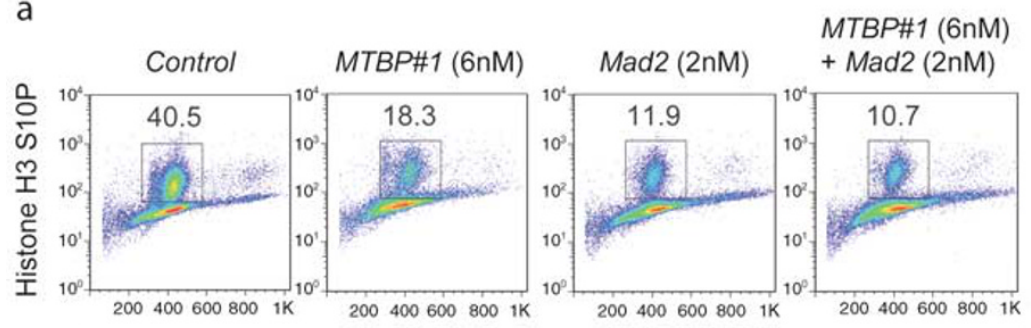

DNA content

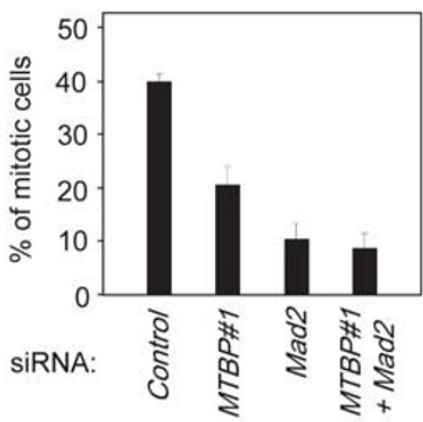

b

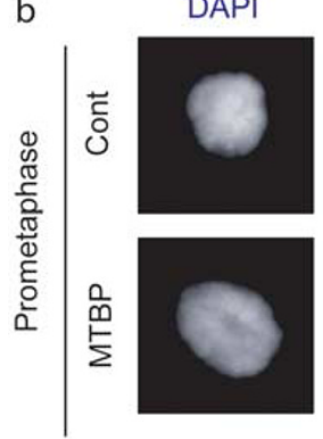

|
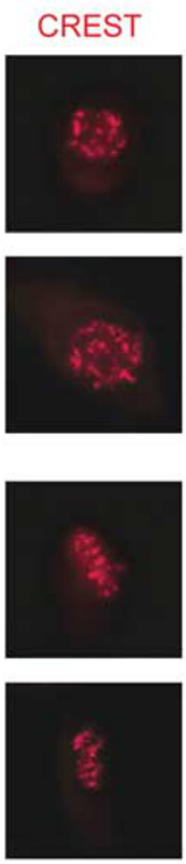

$\operatorname{Mad} 2$
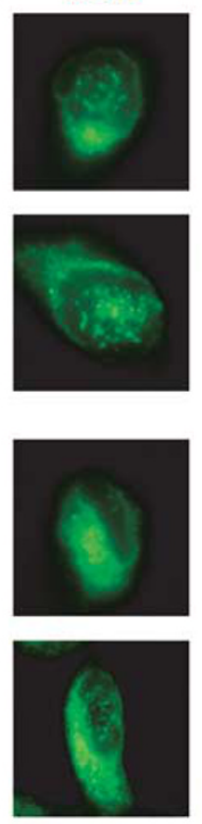
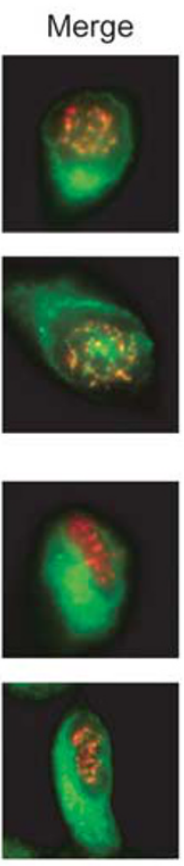
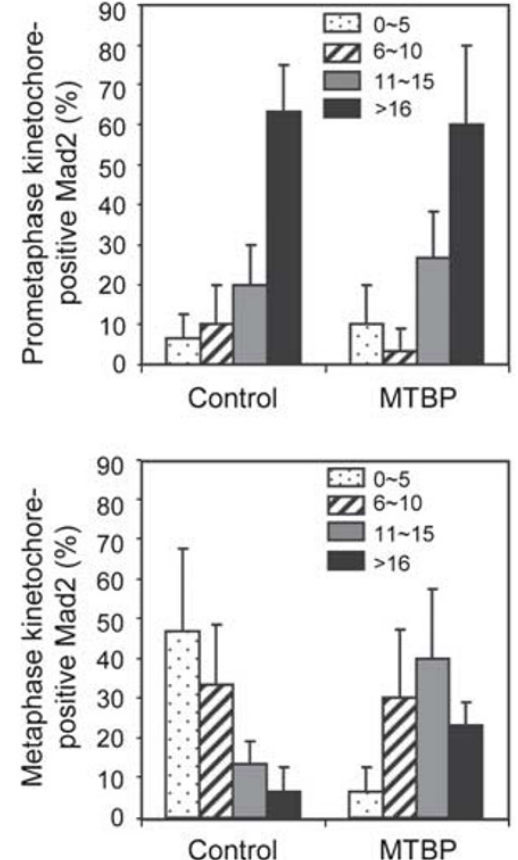

Figure 6 MTBP affects the mitotic checkpoint function via the Mad2 pathway. (a) HeLa cells downmodulated for MTBP and/or Mad2 were examined for the mitotic index. Representative results of flow cytometric analyses with percentages of phospho-histone H3 serine 10 (Histone H3 S1OP)-positive mitotic cells in squares (left) and graph from three independent experiments (right, mean \pm S.D.). (b) Kinetochore localization of Mad2 during prometaphase and metaphase following infection of adenoviral vectors encoding control (Cont) or MTBP (MTBP). HeLa cells were treated with nocodazole for $15 \mathrm{~h}$ after adenoviral infection and released from the treatment for $5 \mathrm{~h}$ to enrich the mitotic cell population. Both attached and unattached cells were fixed and stained for DNA (DAPI, blue), centromere (CREST, red), and Mad2 (green). Based on the numbers of Mad2-staining foci at the kinetochores, cells were categorized into $0-5,6-10,11-15$, and $>16$. Percentages of cells in each category are shown. Representative results (left) and graphs from three independent experiments (right, mean \pm S.D). Merged pictures of the staining results for CREST and Mad2

of Mad2 and MTBP (Supplementary Figure S6). Concomitant downmodulation of MTBP in cells downmodulated for Mad2 did not further reduce the mitotic index to that with Mad2 downmodulation alone (Figure 6a), suggesting that the effect of MTBP on the mitotic checkpoint is at least partially dependent on Mad2.

We additionally examined the effect of MTBP overexpression on the kinetochore localization of Mad2 (Figure 6b). MTBP overexpression did not alter kinetochore localization of Mad2 during prometaphase. However, MTBP overexpressed cells showed more Mad2-staining foci at the metaphase kinetochores than the control (Figure 6b). These results suggest that MTBP overexpression inhibited the release of Mad2 from the kinetochores to the microtubules during metaphase. Taken together, our findings indicate that MTBP is involved in the regulation of the Mad1/Mad2-mediated mitotic checkpoint.

\section{Discussion}

Our results demonstrated that both overexpression and downmodulation of MTBP induced abnormal chromosome segregation and improper mitotic progression. We therefore conclude that MTBP has a crucial role in proper mitotic progression and faithful chromosome segregation.

Increasing evidence indicates that loss of mitotic checkpoint control induces chromosomal instability and tumor development. Both decreased and increased levels of mitotic checkpoint proteins cause a similar cellular response, including improper mitotic progression, chromosome aneuploidy, 
senescence, and tumor development via impaired and overactive mitotic checkpoint activities, respectively. ${ }^{12,18,22,23}$ Therefore, proteins involved in the mitotic checkpoint cannot be simply categorized as either oncogenes or tumor suppressors. Mice null for Mad1, Mad2, BubR1, Bub1, and Bub3 show early embryonic lethal phenotypes because of chromosome missegregation. ${ }^{23-28}$ However, mice heterozygous for Mad1 and Mad2 or mice with the hypomorphic alleles of BubR1 $\left(B u b R 1^{H}\right), B u b 3\left(B u b 3^{H}\right)$, and $B u b 1\left(B u b 1^{H}\right)$ develop either late onset of spontaneous tumors or early aging-associated phenotypes, whereas heterozygous mice for Bub1, Bub3, and BubR1 show no obvious phenotypes. ${ }^{23,27,29-31}$ Importantly, mice overexpressing Mad2 also show accelerated tumorigenesis with chromosomal instability. ${ }^{11,12}$ These results suggest that a threshold level of the mitotic checkpoint-related proteins is necessary for preventing cells from chromosome missegregation, tumor development, and aging-associated phenotypes. Our recent publication demonstrates that complete deletion of MTBP in mice results in early embryonic lethality. ${ }^{3}$ On the other hand, MTBP heterozygous mice show no obvious phenotype but display an enhanced metastatic tumor phenotype when mice are predisposed to tumor development in the $p 53^{+/-}$background. ${ }^{3}$ These results are similar to those of knockout mice for proteins involved in the mitotic checkpoint. Moreover, our results demonstrated that cells with MTBP downmodulation showed similar cellular phenotypes to those with Mad2 and BubR1 downmodulation. Together, these findings support our notion that MTBP positively participates in mitotic checkpoint activity.

Our immunofluorescence results suggest that MTBP is required for the localization of Mad1 and Mad2 at the kinetochores. Central to the formation of the MCC, both Mad1 and Mad2 are recruited to the kinetochores during early prometaphase from the nuclear pore where they are located during interphase. Mad1/Mad2 interactions at the nuclear pore during interphase are important for their kinetochore localization during mitosis. ${ }^{20,21}$ Although multiple regulatory mechanisms have been suggested, little is known about how the Mad1/Mad2 complex is formed at the nuclear pore and how this complex is recruited to the kinetochores. ${ }^{17,32,33}$ Our attempts to demonstrate the effects of MTBP downmodulation on the Mad1/Mad2 interaction and their expression levels were not successful. Additionally, we could not detect the interactions of MTBP with Mad1 and Mad2 (data not shown). However, our results of endogenous MTBP staining demonstrated that a portion of MTBP was localized at the prometaphase kinetochores, and to a much lesser extent at the metaphase kinetochores. Furthermore, cells with MTBP overexpression showed more Mad2-staining foci at the metaphase kinetochores than the control, suggesting that MTBP overexpression inhibited the release of Mad2 from the kinetochores to the microtubules. These results may imply that MTBP has an important role in recruiting and/or retaining the Mad1/Mad2 complex at the kinetochores during prometaphase, but its degradation is required for silencing the mitotic checkpoint during metaphase. It should be noted that the cellular phenotypes induced by MTBP downmodulation in the experiments of chromosome missegregation and mitotic index were not identical with those by Mad2 or BubR1 downmodulation. These results indicate that MTBP is not the only factor that is involved in the mitotic checkpoint regulation and may also suggest that MTBP has other roles in mitotic progression in addition to the regulation of mitotic checkpoint. Thus, several issues, including the exact mechanism by which MTBP participates in mitotic checkpoint, how it affects the localization of Mad1 and Mad2 at the kinetochores, how it contributes to silencing the mitotic checkpoint, and if MTBP has functions other than regulation of Mad1/Mad2 function on mitotic progression, remain open for future research.

In summary, this study illustrates a novel physiological function of MTBP in the regulation of mitotic progression and chromosome segregation, which may facilitate the discovery of new treatment regimens for cancer by targeting mitosis. Our findings may also provide mechanistic insights to previously reported phenotypes associated with altered MTBP expression including cell proliferation, tumor progression, p53 independency, and embryonic lethality of Mtbp-null mice. ${ }^{1,3,4}$

\section{Materials and Methods}

Antibodies. MTBP (K-20 or H-40), cyclin B1 (D-11), Mad2 (17D10), and Mad1 (9B10) antibodies were purchased from Santa Cruz Biotechnology (Santa Cruz, CA, USA). Polyclonal phospho-histone $\mathrm{H} 3$ serine 10 antibody was purchased from Cell Signaling Technology (Danvers, MA, USA). $\alpha$-Tubulin (DM1A), $\beta$-tubulin (D66), and FLAG (M2) antibodies were purchased from Sigma Biochemicals (St. Louis, MO, USA). BubR1 (9/BUBR1) and cyclin A (clone 25) were from BD Biosciences (San Jose, CA, USA). The following antibodies were purchased from the indicated sources: antiserum polyclonal Mad2 (PRB-452C; COVANCE, Princeton, NJ, USA) and human autoimmune CREST serum (Immunovision, Springdale, AR, USA).

Cell culture and synchronization. HeLa cells were obtained from American Type Culture Collection (ATCC). HCT116 p53 ${ }^{+1+}$ and HCT116 p53 ${ }^{-l-}$ cells were kindly provided by Dr. Bert Vogelstein at Johns Hopkins University. To synchronize cells at $\mathrm{G} 1$ phase of the cell cycle, cells were treated with $500 \mu \mathrm{M}$ mimosine (Sigma Biochemicals) for $24 \mathrm{~h}$. Cells were then washed with PBS three times and cultured in fresh media to release them from the early G1 phase of the cell cycle. For prometaphase arrest, cells were treated with $500 \mathrm{ng} / \mathrm{ml}$ nocodazole (Sigma Biochemicals) for $24 \mathrm{~h}$. Mitotic cells were then harvested using the mitotic shake-off method. ${ }^{34}$ To release cells from prometaphase, they were washed with PBS three times and reseeded in fresh media.

Viral vectors and siRNA transfection. An adenoviral vector encoding mouse MTBP was used to overexpress MTBP as described previously. ${ }^{3}$ The control empty adenoviral vector was purchased from Vector Biolabs (Philadelphia, PA, USA). The pCDH-EF1-MCS-T2A-copGFP lentiviral vector (System Biosciences, Mountain View, CA, USA) encoding human MTBP was used for overexpressing MTBP. The siRNA oligonucleotides were chemically synthesized at Dharmacon RNA Technologies (Lafayette, CO, USA). The sequences for siRNAs used in this study were human Mad2 (5'-AAGTGGTGAGGTCCTGGAAAG- $\left.3^{\prime}\right){ }^{35}$ human BubR1 (5'-AACGGGCAUUUGAAUAUGAAA-3'), ${ }^{36}$ human Mad1 (5'-AACAGGCA GTGTCAGCAGAAC- $\left.3^{\prime}\right),{ }^{37}$ and human MTBP\#1 (5'-GCCACAUUGAUUCACU CAGUU- $3^{\prime}$ ). Human MTBP\#2 and control non-target 1 siRNAs were purchased from Santa Cruz Biotechnology and Dharmacon RNA Technologies, respectively. Transfection of siRNA was performed using HiPerFect (QIAGEN, Valencia, CA, USA) according to the manufacturer's instructions.

Western blotting. Cells were lysed with RIPA buffer $(50 \mathrm{mM}$ Tris- $\mathrm{HCl}$ $\mathrm{pH} 7.6,150 \mathrm{mM} \mathrm{NaCl}, 1 \mathrm{mM}$ EDTA, $1 \%$ sodium deoxycholate, $0.1 \%$ Triton $\mathrm{X}-100$, and $0.1 \%$ sodium dodecyl sulfate (SDS)) supplemented with protease inhibitor cocktail (Roche, Indianapolis, IN, USA), $1 \mathrm{mM}$ phenylmethylsulfonyl fluoride (PMSF), $0.2 \mathrm{mM}$ sodium orthovanadate, and $100 \mathrm{mM}$ sodium fluoride. Whole-cell extracts were separated by SDS-PAGE and transferred onto PVDF membranes (GE Healthcare Biosciences, Piscataway, NJ, USA). After blocking with $5 \%$ non-fat milk in $1 \times$ Tris-buffered saline (TBS) with $0.1 \%$ Tween-20 (TBS-T), blots were incubated with primary antibodies, and then with secondary antibodies conjugated with horseradish peroxidase (Thermo Scientific, Waltham, MA, USA). To visualize 
signals, Super Signal West Dura or Femto Chemiluminescent substrates (Pierce Biotechnology, Rockford, IL, USA) were used according to the manufacturer's instructions. The signals were detected using a Bio-Rad VersaDoc imaging system (Bio-Rad, Hercules, CA, USA) or KODAK X-ray developer.

Quantitative reverse transcriptase (RT)-PCR by TaqMan assays. RNeasy (Qiagen) and first-strand cDNA Synthesis Kit (GE Healthcare) were used for RNA isolation and reverse transcriptase reactions, respectively. TaqMan assays were performed according to the manufacturer's specifications (Applied Biosystems, Foster City, CA, USA) using the following probes: Gapdh (Hs99999905_m1) and MTBP (Hs00608686_m1). Relative mRNA expression was normalized to the value of Gapdh for each reaction.

Protein stability study. After HeLa cells were cultured in the presence of nocodazole for $24 \mathrm{~h}$, mitotic cells were harvested by the mitotic shake-off method. After washing with PBS, cells were reseeded in fresh media containing $100 \mu \mathrm{g} / \mathrm{ml}$ $\mathrm{CHX}$. Cells were harvested at the indicated time points and subjected to western blotting for endogenous MTBP, cyclin B1, and $\beta$-tubulin.

Co-immunoprecipitation studies. Cells were lysed with RIPA lysis buffer containing protease inhibitor cocktail. Approximately $200 \mu \mathrm{g}$ of whole-cell lysates were incubated with protein-specific antibodies overnight at $4{ }^{\circ} \mathrm{C}$, followed by precipitation of the antibody-protein complex using protein $A / G$ plus-agarose (Santa Cruz Biotechnology). In each immunoprecipitation experiment, an isotype negative control was used. After washing with RIPA buffer, precipitates were analyzed by western blotting.

Immunofluorescence study. Cells were plated onto poly-D-lysine/laminincoated glass coverslips (BD Biosciences). Cells were fixed and permeabilized with $4 \%$ formaldehyde in $100 \mathrm{mM}$ PIPES (pH 6.8), $10 \mathrm{mM} \mathrm{EGTA}, 1 \mathrm{mM} \mathrm{MgCl}$, and $0.2 \%$ Triton-X 100 for $15 \mathrm{~min}$ at room temperature. Following PBS washing, cells were blocked in 1\% BSA in PBS plus $0.1 \%$ Tween-20 (PBS-T) for 30 min and further incubated with primary antibodies for $60 \mathrm{~min}$. After PBS-T washing, cells were incubated with the appropriate secondary antibodies. Samples were mounted in the ProLong Gold Antifade Reagent with DAPI (Invitrogen, Carlsbad, CA, USA), and results were analyzed with a Leica epifluorescence microscope (Leica, Bannockburn, IL, USA).

For the kinetochore localization studies, cells were fixed with freshly prepared $1 \%$ paraformaldehyde in PBS for 20 min. Cells were then permeabilized with $0.2 \%$ Triton-X 100 in PBS (PBS-T) for 3 min, and were blocked in 3\% BSA in PBS-T for $30 \mathrm{~min}$, followed by incubation with appropriate antibodies. ${ }^{38,39}$ To detect kinetochore MTBP, H-40 antibody (Santa Cruz Biotechnology) was used. Z-sectioned images were obtained on a Zeiss Axioplan II deconvolution microscope (Carl Zeiss, Thornwood, NY, USA) and processed as described by Guenther et al. ${ }^{40}$

Flow cytometric analysis. For cell cycle analysis, cells fixed with $70 \%$ ethanol at $-20^{\circ} \mathrm{C}$ were stained with $\mathrm{PI}$ solution (Invitrogen) in the presence of $10 \mu \mathrm{g} / \mathrm{ml}$ RNase, followed by flow cytometric analysis using FACSCalibur (BD Biosciences). To measure the mitotic index, cells were stained with a phosphohistone $\mathrm{H} 3$ Serine 10 antibody. All data were analyzed using the FlowJo software (Tree Star, Ashland, OR, USA).

Colony outgrowth assays. The colony outgrowth assays were performed as described previously with a minor modification. ${ }^{10}$ At 2 days after transfection of siRNAs into HeLa cells, cells were incubated with either $500 \mathrm{ng} / \mathrm{ml}$ of nocodazole or DMSO (solvent control). After $24 \mathrm{~h}$ of incubation, both attached and floating cells were harvested and washed with PBS. Following Trypan blue staining, live cells (2000) were seeded onto $6 \mathrm{~cm}$ dish in triplicate and allowed to grow for 12-14 days. Cells were then stained with crystal violet, and colonies were counted. The percentage of colonies formed was determined by normalizing to the number of colonies formed by DMSO-treated control siRNA-transfected cells.

Cell proliferation assays. At $48 \mathrm{~h}$ after siRNAs transfection into HeLa or HCT116 cells, cells $\left(2 \times 10^{4}\right)$ were reseeded onto six-well plates in triplicate (day 0$)$. Cell numbers were counted at 2, 4, 6, 7, 8, and 9 days after reseeding.

SA- $\beta$-gal staining. At $48 \mathrm{~h}$ after siRNA transfection, cells $\left(1 \times 10^{4}\right)$ were reseeded on 24-well plates. After 7 days, they were fixed for $10 \mathrm{~min}$ in $3.7 \%$ formaldehyde, washed with PBS, and stained overnight at $37^{\circ} \mathrm{C}$ with $\mathrm{X}$-gal staining solution containing $40 \mathrm{mM}$ citric acid/sodium phosphate $\mathrm{pH} 6.0,5 \mathrm{mM}$ potassium ferricyanide, $5 \mathrm{mM}$ potassium ferrocyanide, $150 \mathrm{mM} \mathrm{NaCl}, 2 \mathrm{mM} \mathrm{MgCl}_{2}$, and $1 \mathrm{mg} / \mathrm{ml} \mathrm{X-gal.}$

Live cell time-lapse imaging and analysis. Media was exchanged with dye-free Leibovitz's L-15 medium (Invitrogen) supplemented with 10\% fetal bovine serum and the cells were processed for time-lapse imaging. Cells stably expressing histone H2B-eGFP or H2B-mCherry were imaged in a stage-top incubator that was maintained at $37^{\circ} \mathrm{C}$. Images were acquired every $3 \mathrm{~min}$ for $6-12 \mathrm{~h}$ using a $\times 20$ or $\times 40$ PLANAPO numerical aperture 0.55 objective on Zeiss Axiovert 200M inverted microscope (Carl Zeiss) equipped with a Zeiss AxioCam MRm camera. The time-lapse imaging system was controlled by AxioVs40 4.6.3.0 software. Time-lapse image stacks were converted to JPEG and analyzed for mitotic progression and duration of different mitotic phases.

Statistical analysis. Experiments were performed independently at least three times with values expressed as mean \pm S.D. Statistical analysis was performed with the analysis of variance (one-way ANOVA) between groups using the statistical software KaleidaGraph (version 3.6; Abelbeck/Synergy Software, Reading, PA, USA). When significance was detected, data were further analyzed using the Bonferroni's multiple comparison test. In each analysis, values of $P<0.05$ were considered to be statistically significant.

\section{Conflict of interest}

The authors declare no conflict of interest.

Acknowledgements. We thank Drs. Guillermina Lozano, Terry Van Dyke, Christine M Eischen, Jay K Kolls, Wanguo Liu, and Ryoko Kibe for helpful discussion, and Drs. Becky Worthylake and James F Guenther for technical assistance. We also thank Mr. Byron M Wood, Ms. Christina S Chen, Ms. Kristy-Le T Nguyen, Swathi lyer, and Dr. Britt Bromberg for editing the manuscript. This work was supported by grants from P20 RR020152-02 (PLD), RSG-09-169-01-CSM (TI), and LCRC start-up (TI).

1. Boyd MT, Vlatkovic N, Haines DS. A novel cellular protein (MTBP) binds to MDM2 and induces a G1 arrest that is suppressed by MDM2. J Biol Chem 2000; 275: 31883-31890.

2. Brady M, Vlatkovic N, Boyd MT. Regulation of p53 and MDM2 activity by MTBP. Mol Cell Biol 2005; 25: 545-553.

3. Iwakuma T, Tochigi Y, Van Pelt CS, Caldwell LC, Terzian T, Parant JM et al. Mtbp haploinsufficiency in mice increases tumor metastasis. Oncogene 2008; 27: 1813-1820.

4. Odvody J, Vincent T, Arrate MP, Grieb B, Wang S, Garriga J et al. A deficiency in Mdm2 binding protein inhibits Myc-induced B-cell proliferation and lymphomagenesis. Oncogene 2010; 29: 3287-3296.

5. Cleveland DW, Mao Y, Sullivan KF. Centromeres and kinetochores: from epigenetics to mitotic checkpoint signaling. Cell 2003; 112: 407-421.

6. Suijkerbuijk SJ, Kops GJ. Preventing aneuploidy: the contribution of mitotic checkpoint proteins. Biochim Biophys Acta 2008; 1786: 24-31.

7. Baker DJ, Chen J, van Deursen JM. The mitotic checkpoint in cancer and aging: what have mice taught us? Curr Opin Cell Biol 2005; 17: 583-589.

8. Kops GJ, Weaver BA, Cleveland DW. On the road to cancer: aneuploidy and the mitotic checkpoint. Nat Rev Cancer 2005; 5: 773-785.

9. Williams GL, Roberts TM, Gjoerup OV. Bub1: escapades in a cellular world. Cell Cycle 2007; 6: 1699-1704.

10. Niikura $Y$, Dixit A, Scott R, Perkins G, Kitagawa K. BUB1 mediation of caspaseindependent mitotic death determines cell fate. J Cell Biol 2007; 178: 283-296.

11. Schvartzman JM, Sotillo R, Benezra R. Mitotic chromosomal instability and cancer: mouse modelling of the human disease. Nat Rev Cancer 2010; 10: 102-115.

12. Sotillo R, Hernando E, Diaz-Rodriguez E, Teruya-Feldstein J, Cordon-Cardo C, Lowe SW et al. Mad2 overexpression promotes aneuploidy and tumorigenesis in mice. Cancer Cell 2007; 11: 9-23

13. Sotillo R, Schvartzman JM, Socci ND, Benezra R. Mad2-induced chromosome instability leads to lung tumour relapse after oncogene withdrawal. Nature 2010; 464: 436-440.

14. Niikura $\mathrm{Y}$, Ogi $\mathrm{H}$, Kikuchi K, Kitagawa K. BUB3 that dissociates from BUB1 activates caspase-independent mitotic death (CIMD). Cell Death Differ 2010; 17: 1011-1024.

15. Fung MK, Cheung HW, Wong HL, Yuen HF, Ling MT, Chan KW et al. MAD2 expression and its significance in mitotic checkpoint control in testicular germ cell tumour. Biochim Biophys Acta 2007; 1773: 821-832.

16. Qi W, Martinez JD. Reduction of 14-3-3 proteins correlates with increased sensitivity to killing of human lung cancer cells by ionizing radiation. Radiat Res 2003; 160: 217-223. 
17. Diaz-Rodriguez E, Sotillo R, Schvartzman JM, Benezra R. Hec1 overexpression hyperactivates the mitotic checkpoint and induces tumor formation in vivo. Proc Nat Acad Sci USA 2008; 105: 16719-16724.

18. Rao CV, Yamada HY, Yao Y, Dai W. Enhanced genomic instabilities caused by deregulated microtubule dynamics and chromosome segregation: a perspective from genetic studies in mice. Carcinogenesis 2009; 30: 1469-1474.

19. Perez de Castro I, de Carcer G, Malumbres M. A census of mitotic cancer genes: new insights into tumor cell biology and cancer therapy. Carcinogenesis 2007; 28: 899-912.

20. Campbell MS, Chan GK, Yen TJ. Mitotic checkpoint proteins HsMAD1 and HsMAD2 are associated with nuclear pore complexes in interphase. J Cell Sci 2001; 114: 953-963.

21. Chung $E$, Chen RH. Spindle checkpoint requires Mad1-bound and Mad1-free Mad2. Mol Biol Cell 2002; 13: 1501-1511.

22. Gjoerup OV, Wu J, Chandler-Militello D, Williams GL, Zhao J, Schaffhausen B et al. Surveillance mechanism linking Bub1 loss to the p53 pathway. Proc Natl Acad Sci USA 2007; 104: 8334-8339.

23. Michel LS, Liberal V, Chatterjee A, Kirchwegger R, Pasche B, Gerald W et al. MAD2 haploinsufficiency causes premature anaphase and chromosome instability in mammalian cells. Nature 2001; 409: 355-359.

24. Dobles M, Liberal V, Scott ML, Benezra R, Sorger PK. Chromosome missegregation and apoptosis in mice lacking the mitotic checkpoint protein Mad2. Cell 2000; 101: 635-645.

25. Kalitsis P, Fowler KJ, Griffiths B, Earle E, Chow CW, Jamsen K et al. Increased chromosome instability but not cancer predisposition in haploinsufficient Bub3 mice. Genes Chromosomes Cancer 2005; 44: 29-36.

26. Dai W, Wang Q, Liu T, Swamy M, Fang $Y$, Xie S et al. Slippage of mitotic arrest and enhanced tumor development in mice with BubR1 haploinsufficiency. Cancer Res 2004; 64: $440-445$

27. Jeganathan K, Malureanu L, Baker DJ, Abraham SC, van Deursen JM. Bub1 mediates cell death in response to chromosome missegregation and acts to suppress spontaneous tumorigenesis. J Cell Biol 2007; 179: 255-267.

28. Perera D, Tilston V, Hopwood JA, Barchi M, Boot-Handford RP, Taylor SS. Bub1 maintains centromeric cohesion by activation of the spindle checkpoint. Dev Cell 2007; 13: 566-579.
29. Baker DJ, Jeganathan KB, Cameron JD, Thompson M, Juneja S, Kopecka A et al. BubR1 insufficiency causes early onset of aging-associated phenotypes and infertility in mice. Nat Genet 2004; 36: 744-749.

30. Baker DJ, Jeganathan KB, Malureanu L, Perez-Terzic C, Terzic A, van Deursen JM. Early aging-associated phenotypes in Bub3/Rae1 haploinsufficient mice. J Cell Biol 2006; 172: $529-540$

31. Schliekelman M, Cowley DO, O'Quinn R, Oliver TG, Lu L, Salmon ED et al. Impaired Bub1 function in vivo compromises tension-dependent checkpoint function leading to aneuploidy and tumorigenesis. Cancer Res 2009; 69: 45-54.

32. Quimby BB, Arnaoutov A, Dasso M. Ran GTPase regulates Mad2 localization to the nuclear pore complex. Eukaryot Cell 2005; 4: 274-280.

33. Yang M, Li B, Tomchick DR, Machius M, Rizo J, Yu H et al. p31comet blocks Mad2 activation through structural mimicry. Cell 2007; 131: 744-755.

34. Schorl C, Sedivy JM. Analysis of cell cycle phases and progression in cultured mammalian cells. Methods 2007; 41: 143-150.

35. Yun M, Han YH, Yoon SH, Kim HY, Kim BY, Ju YJ et al. p31comet induces cellular senescence through p21 accumulation and Mad2 disruption. Mol Cancer Res 2009; 7: 371-382.

36. Johnson VL, Scott MI, Holt SV, Hussein D, Taylor SS. Bub1 is required for kinetochore localization of BubR1, Cenp-E, Cenp-F and Mad2, and chromosome congression. J Cell Sci 2004; 117: 1577-1589.

37. Luo X, Tang Z, Rizo J, Yu H. The Mad2 spindle checkpoint protein undergoes similar major conformational changes upon binding to either Mad1 or Cdc20. Mol Cell 2002; 9: 59-71.

38. Skoufias DA, Andreassen PR, Lacroix FB, Wilson L, Margolis RL. Mammalian mad2 and bub1/bubR1 recognize distinct spindle-attachment and kinetochore-tension checkpoints. Proc Natl Acad Sci USA 2001; 98: 4492-4497.

39. Lee SH, Sterling H, Burlingame A, McCormick F. Tpr directly binds to Mad1 and Mad2 and is important for the Mad1-Mad2-mediated mitotic spindle checkpoint. Genes Dev 2008; 22: 2926-2931.

40. Guenther JF, Cameron JE, Nguyen HT, Wang Y, Sullivan DE, Shan B et al. Modulation of lung inflammation by the Epstein-Barr virus protein Zta. Am J Physiol Lung Cell Mol Physiol 2010; 299: L771-L784.

\section{Supplementary Information accompanies the paper on Cell Death and Differentiation website (http://www.nature.com/cdd)}

